\title{
Application of intelligent image processing in the construction material industry
}

\author{
Katharina Anding ${ }^{1}$, Daniel Garten ${ }^{2}$ and Elske Linß ${ }^{3}$ \\ ${ }^{1}$ Faculty of Mechanical Engineering, Department of Quality Assurance and Industrial Image Processing, Ilmenau University of Technology, \\ IImenau, Germany \\ ${ }^{2}$ GFE - society for production engineering and development Schmalkalden, Germany \\ ${ }^{3}$ Faculty Civil Engineering, Chair Material of Building, Bauhaus-University of Weimar, Weimar, Germany
}

\section{ABSTRACT}

This paper discusses two analysis activities in the construction material industry, which could be solved by intelligent image processing algorithms. The first task is the optical identification of recycled aggregates of construction and demolition waste (CDW) as basis of an innovative sorting method on the field of processing of CDW. The second and far more complicated task due to very high phenotypical object variabilities within the subclasses is the optical analysis of samples from mineral aggregates. The application of automatically optical identification methods can save time and thus costs. This is an important factor for small and medium-sized companies in the construction material industry.

\begin{abstract}
Keywords: optical identification, machine learning, construction and demolition waste (CDW), mineral aggregates
Citation: Katharina Anding, Daniel Garten and Elske Linß, "Application of intelligent image processing in the construction material industry", Acta IMEKO, vol. 2, no. 1, article 14, August 2013, identifier: IMEKO-ACTA-02(2013)-01-14
\end{abstract}

Editors: Paolo Carbone, University of Perugia, Italy; Gerhard Linß, Ilmenau University of Technology, Germany

Received April $2^{\text {nd }}, 2013$; In final form April $4^{\text {th }}, 2013$; Published August 2013

Copyright: @ 2013 IMEKO. This is an open-access article distributed under the terms of the Creative Commons Attribution 3.0 License, which permits unrestricted use, distribution, and reproduction in any medium, provided the original author and source are credited

Funding: This work was supported by German Federal Ministry of Economics and Technology (BMWI)

Corresponding author: Katharina Anding, e-mail: katharina.anding@tu-ilmenau.de

\section{INTRODUCTION}

The industry of building materials is one of the biggest material flows in Germany and also the demolition of buildings causes very huge masses of construction and demolition waste (CDW). The construction materials industry is known as a very conservative branch of trade. Most of the used quality tests are based on simple methods. Especially the sorting of materials is done by mechanical methods, which are limited in the sorting of complex material mixes. The separation or identification of different materials is done very often by human hand sorting rather than by automatic inspection systems. For the production of concrete the used aggregates have to meet the directive's requirements. It has to be decided in the production of recycling "green" concrete and the production of standard concrete, so that two typical analysis tasks in these sectors are given:

- Optical identification of recycled aggregates of construction and demolition waste (CDW) for the production of recycling "green" concrete and

- Optical analysis of mineral aggregates used as primary concrete aggregate.
The aim of the described investigations is to form the basis for the development of standard systems to solve problems in the field of quality assurance of construction material. These systems could also be used by small companies as opposed to expensive custom solutions.

Before describing the research results in the two mentioned application areas some of the theory behind the used algorithms will be explained. The work is focussed on feature extraction, feature selection and design of the classifier model. The whole process is also known as the pattern recognition chain.

\section{THEORY BEHIND THE USED ALGORITHMS}

\subsection{Feature Extraction}

After segmentation and transformation from the RGB to HSI colour space a feature vector for every object has been calculated. Colour and texture features were used like the mean value per channel and features calculated from the cooccurrence matrix like energy, homogeneity and contrast per each HSI-channel. Scale and rotation-invariant shape features were also calculated like modified Fourier descriptors. 
The used feature algorithms are part of the machine vision software Halcon and were described in the release notes for MVTec HALCON 8.0.3 [1]. Here three of the used feature algorithms are described in detail. The feature operator circularity $(s)$ calculates the similarity of the input region of the given image with a circle. If $F$ is the area of the image region and max is the maximum distance from the center point to all contour pixels, the shape factor $s$ is calculated as [1]

$$
s=\frac{F}{\left(\max ^{2} \cdot \pi\right)} .
$$

If the region is a circle then the shape factor $s$ equals the value 1 . If the region has another shape as a circle, $s$ is smaller than 1.

Another feature operator is the entropy and anisotropy coefficient of the image, defined as [1]

$$
\begin{aligned}
& \text { entropy }=-\sum_{0}^{255} r e l[i] * l d(r e l[i]), \\
& \text { anisotropy }=\frac{\sum_{0}^{k} r e l[i] * l d(r e l[i])}{\text { entropy }} .
\end{aligned}
$$

The used parameters are:

$$
\begin{aligned}
& r e[[i] \text { - histogram of relative gray value frequencies, } \\
& I \quad \text { - gray value of input image }(0 . .255) \text { and } \\
& k \quad \text { - the smallest possible gray value. }
\end{aligned}
$$

\subsection{Best differentiating features - feature selection}

The performance of several classification algorithms decreases by using redundant and irrelevant features in the feature vector. In addition the calculation needs a lot of time for an excessive feature vector with non-informative features. But the aim of the investigations is a real-time recognition system of building materials.

For example the time a Support Vector Machine (SVM) needs for classification depends linearly on the dimension of the feature vector and number of support vectors. It is not expedient to calculate irrelevant features. In summary the aim is the reduction of the dimension of the feature vector.

Filter selection methods are independent of any classifiers [2]. They filter out features with low discriminatory power. These methods are based on the performance evaluation metric calculated directly from the given dataset [2]. In contrast to wrapper methods, filter methods are normally not computationally intensive.

Wrapper methods are very time-consuming with using a complex training algorithm like the SVM. This fact rests in estimating the discriminatory power of features by calculating the recognition accuracy of each feature selection (feature subset).

So filter selection methods were used, especially the InfoGain-Attribute-Evaluator and Chi-Squared-Attribute-Evaluator.

The Information Gain measure is based on the entropy [3]. The entropy for the class distribution $C$ is evaluated as

$$
H(C)=\sum_{c \in C} p(c) \cdot \log _{2} \cdot p(c)
$$

The conditional entropy for class $c$ and feature $F$ is evaluated according to the following formula,

$$
H(C \mid F)=\sum_{f \in F} p(f) \sum_{c \in C} p(c \mid f) \cdot \log _{2} \cdot p(c \mid f) \text {. }
$$

From this it follows that the Info Gain for the specific feature $i$ is evaluated in the form of

$$
I G_{i}=H(C)-H\left(C \mid F_{i}\right) .
$$

\begin{tabular}{|c|c|c|c|c|c|}
\hline $\begin{array}{l}\text { distance-based } \\
\text { classifiers }\end{array}$ & $\begin{array}{l}\text { geometric } \\
\text { classifiers }\end{array}$ & $\begin{array}{l}\text { decision tree } \\
\text { classifiers }\end{array}$ & $\begin{array}{l}\text { statistical } \\
\text { classifiers }\end{array}$ & $\begin{array}{l}\text { support vector } \\
\text { machines }\end{array}$ & $\begin{array}{l}\text { artificial neural } \\
\text { network }\end{array}$ \\
\hline $\begin{array}{l}\text { - k-nearest } \\
\text { neighbour } \\
\text { algorithm, } \\
\text { • minimum } \\
\text { distance } \\
\text { algorithm, } \\
\text { - Mahalanobis } \\
\text { algorithm }\end{array}$ & $\begin{array}{c}\text { - hyperbox } \\
\text { classifier }\end{array}$ & $\begin{array}{l}\text { - CART, } \\
\text { - ID3, } \\
\text { - C4.5, } \\
\text { - random } \\
\text { forest }\end{array}$ & $\begin{array}{l}\text { - Bayes } \\
\text { classifier, } \\
\text { - Naive-Bayes } \\
\text { classifier, } \\
\text { - Maximum- } \\
\text { Likelihood }\end{array}$ & $\begin{array}{l}\text { - C-SVM, } \\
\text { - v-SVM, } \\
\text { - LibSVM } \\
\rightarrow \text { different } \\
\text { kernel functions: } \\
\text { linear, } \\
\text { polynomial, } \\
\text { sigmoid, } \\
\text { radial basis } \\
\text { function }\end{array}$ & $\begin{array}{l}\text { - multi-layer } \\
\text { perceptron } \\
\text { - learning } \\
\text { vector } \\
\text { quantization }\end{array}$ \\
\hline
\end{tabular}

As a result a score is calculated for each single feature. A statement of the specific discriminatory power is represented by this score.

The Chi-Squared filter method estimates the distributional properties of a statistical basic population in consideration of a specific distributional property [2]. Setino et al. [4] found out that the discretization is an appropriate instrument for selection of numerical features.

Figure 1. Classifiers of supervised learning [6]. 
The discretization is carried out by using the Chi-Squared statistic. The Chi-Squared value has to be determined as the test statistic for a significance test.

\subsection{Supervised machine learning - used classifiers}

There are a plurality of classifiers in the field of supervised learning. In the theory of machine learning Naive Bayes classifier, decision trees, k-nearest-neighbour algorithms, neural networks and SVM are common classifiers [5]. An overview of supervised learning algorithms is shown in Figure 1 [6].

Different classification algorithms of the machine learning library Weka [7] were tested after feature extraction for the problem of CDW recognition, such as LibSVM, Random Forest, k-nearest neighbour, Naive Bayes and J48 by using a 10 -fold cross validation. For the optical analysis of mineral aggregates we focussed on the Halcon-SVM.

Especially the classification with SVM needs an optimal parameter selection for a good classification performance. First an introduction to the characteristic of SVM is given in this paper. The SVM was introduced by [8] and is mentioned as being one of the most powerful classifiers today. It is derived from the statistical learning theory [9]. The algorithm is motivated by the structural risk minimization, which says that not only the training error but also the complexity of the model influences its generalization ability. The SVM was designed to solve binary classification problems but there are different strategies to solve multi-class problems, too. The SVM executes a non-linear projection of data in a higher dimensional feature space. The classes are separable in a linear way in this higher dimensional feature space. During the training process an optimal hyperplane is constructed. Optimal means that it leaves a maximal margin between the hyperplane and the closest training point on both sides. The kernel function $k\left(x, x_{i}\right)$ extends the linear discriminant SVM to a nonlinear machine. The given decision function is [2]

$$
f(x)=\operatorname{sgn}\left(\sum_{i=1}^{m} \alpha_{i} y_{i} k\left(x, x_{i}\right)+b\right) .
$$

The radial basis function kernel (rbf) is one of the popular kernel functions and is defined as

$$
k\left(x, x^{\prime}\right)=e^{-\gamma\left\|x-x^{\prime}\right\|^{2}} .
$$

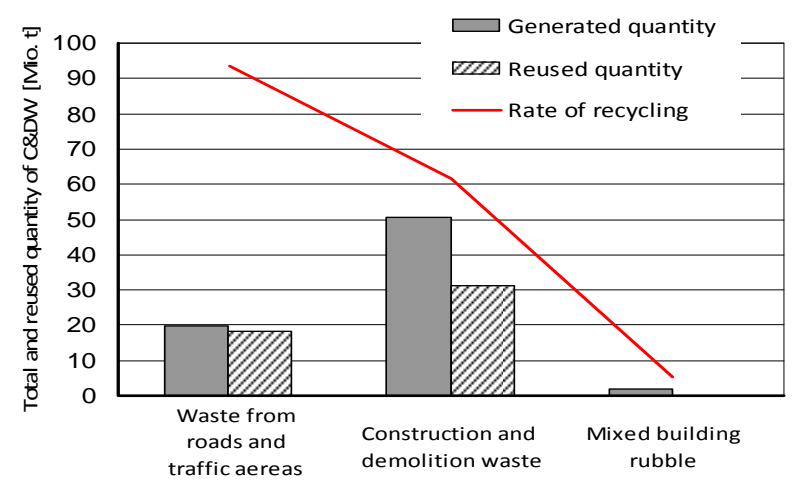

Figure 2. Reuse of different kinds of CDW.

\section{RECOGNITION OF CONSTRUCTION AND DEMOLITION WASTE (CDW)}

\subsection{Introduction}

Construction and demolition waste (CDW) are the biggest waste flow in Germany. There was an amount of 84.5 million tons of CDW in the year 2008 [10]. The recycling rate amounts to $80 \%$ (67 million tons). Certainly the recycling rate depends on the composition and heterogeneity of material (Figure 2).

A significant decrease can be observed in the recycling rate with increasing heterogeneity of the recycled material.

For recycled masonry aggregates and recycled mixed aggregates the lowest recycling rates are found because of the high heterogeneity and the mineral admixtures. Therefore the reuse of these materials is very difficult.

Regarding to the application of C\&D aggregates, most of them are used in road pavements and earth works, not really substituting the natural aggregate applications. Only a very small part of around 5\% flows back in the production of recycling concrete.

The down cycling scenario is also common in other countries [11], [12]. It is not considered sustainable because the use of land will be critical in the future and land filling should be avoided [13].

The recycling industry of building materials is dominated by simple technologies. For instance the single-stage crushing is used with advance sieving and separation of reinforcement steel by over belt magnetic separator. For the processing of building materials sorting processes are only used for the separation of light components until now.

These technologies are not able to separate the incidental mixed aggregates. They are suitable in no way for "new building materials" including connected building materials, which will be used more and more in the building industry.

CDW from building constructions are heterogeneous mixtures of brick, mineral bounded building materials (concrete, sand-lime-brick, aerated concrete, lightweight concrete), mortar, plaster, insulation material, wood and plastic etc. Sorting analyses of crushed CDW confirm the variety in recycling materials. Analyses of the density show a large range of bulk density. The water adsorption is much higher and the grain strength lower than for natural aggregates. The low quality of C\&D aggregates and their variability seems to be the most important aspect to limit its application in concrete. In fact, the composition and physical properties of $\mathrm{C} \& \mathrm{D}$ aggregates are variable in a wide range.

The heterogeneity prevents the profitable reuse. Therefore it is necessary to reduce the heterogeneity. But this is possible only by a multi-stage process with several classification and sorting steps.

It is indispensable to separate the CDW mixtures to establish a reliable and demanding reuse. This is the basis for the development of specific products which are based on the characteristic properties of the materials. And it is also the basis for the return of pure material as secondary material in the production of primary material.

The aim is the realization of real closed cycles and a high standard of quality in recycling.

As in other sectors of recycling, for example the glass or plastic recycling, the sensor-based sorting has become more interesting in the recycling of building materials and sorting of 
minerals in the last years. They mainly use optical, magnetic, NIR or X-Ray sensors.

The application of automatic sensor sorting in the areas of mining and recycling is successful in Europe and will increase in the following years. The benefits are the increase of the end product value and the cost reduction of downstream handling steps in the processing [14], [15].

The first investigations are focussed on the optical differentiation of phenotypically similar building materials like concrete, aerated concrete, lightweight concrete and also porous and dense brick. First investigations were done on new, not used building materials, which were crushed.

An optical solution for determination of building material classes was investigated by using methods of image processing and machine learning. Several optical attributes were found, which have discriminatory power to classify the chosen materials.

Several classification algorithms of supervised machine learning were tested on different feature vectors as a numerical representation of objects of the given dataset. The different feature vectors were built by using feature selection methods, especially filter methods like Information Gain [3] and Chi Squared [2], [4]. As a result the best differentiating features and the most qualified classifiers were attained for solving this optical identification task of building materials.

\subsection{Realization of investigations}

A precondition for a satisfying technical performance of the automated analysis is a good analyzable image. Images of 35 different material samples were taken (8 concretes, 7 aerated concretes, 9 lightweight concretes and 3 bricks). The samples were captured by a RGB matrix camera (see Figure 3). A combination of incident and transmitted light was chosen for capturing colour images. The lighting device consists of three LED-light lines and a light table to visualize the specific class in an optimal way. All images were taken under constant conditions.

A blue foil was installed on the light table to separate the particles as good as possible from the background. Almost 1000 images of particles out of each material class were taken to realize a good statistical comparison. It means that more than 100 particles per sample were captured. The particle sizes were
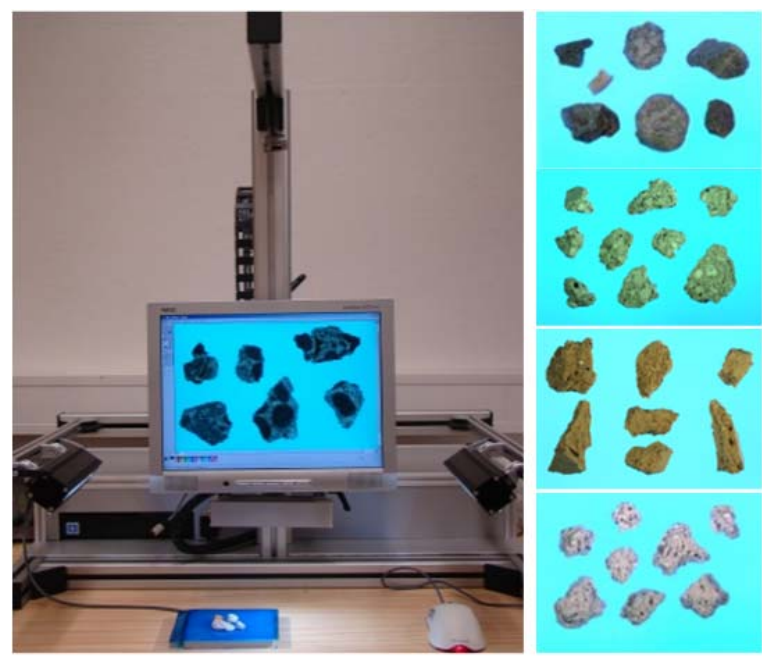

a.)

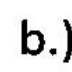

.)

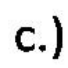

d.)

Figure 3. Image system "QI Inspector" [19] and images of samples a) concrete, b) lightweight concrete, c) brick and d) aerated concrete. in the range of 4 to $8 \mathrm{~mm}$.

Table 1 shows a selection of the best ranked texture and contour features by using the Info Gain method.

As a result the colour features and in particular texture features are the best discriminating features for the given problem, far ahead of contour features. The most useful texture features can be calculated in the H- and S-channel of the HSI colour space. Independent of the two selection methods, nearly the same ranking list was calculated with similar ranking positions. The ranking list of features was used to build up different feature subsets with the number of best ranked features. This means, when the number of used best ranked features equals 16 , the 16 best ranked features of the ranking list were used to build up a feature subset. Its discriminatory power is specified by determining the classification accuracy of a trained and tested classifier. Finally there is a statement given how many best ranked features are needed for reaching a good classification performance.

In the investigations the LibSVM [16] of Weka was tested with the rbf-kernel and different parameter modifications for

Table 1. Selection of the best Info-Gain ranked features.

\begin{tabular}{|c|c|c|c|c|}
\hline & feature name & channel & description [8] & ranking pos. \\
\hline \multirow{10}{*}{ 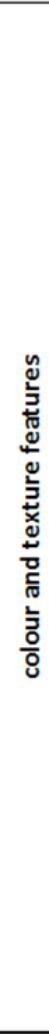 } & $\begin{array}{l}\text { Fit_Surface_2nd_ } \\
\text { order_Zeta }\end{array}$ & $\mathrm{s}$ & $\begin{array}{l}\text { calculates the gray value moments and } \\
\text { the parameters of the approximation of } \\
\text { the gray values by a second order } \\
\text { surface, zeta is the most useful } \\
\text { parameter }\end{array}$ & 1 \\
\hline & $\begin{array}{l}\text { Cooc_Feat__ } \\
\text { Energy }\end{array}$ & H & $\begin{array}{l}\text { calculates the gray value feature energy } \\
\text { from cooc-matrix, energy is a measure of } \\
\text { homogeneity of the gray values }\end{array}$ & 2 \\
\hline & Fuzzy_Entropy1 & $\mathrm{s}$ & $\begin{array}{l}\text { calculates the fuzzy entropy of a fuzzy } \\
\text { set, measure of how well the image } \\
\text { approximates a white or black image }\end{array}$ & 3 \\
\hline & $\begin{array}{l}\text { Fit_Surface_2nd_ } \\
\text { order_Zeta }\end{array}$ & H & $\begin{array}{l}\text { calculates the gray value moments and } \\
\text { the parameters of the approximation of } \\
\text { the gray values by a second order } \\
\text { surface, zeta is the most useful } \\
\text { parameter }\end{array}$ & 4 \\
\hline & $\begin{array}{l}\text { Moments_Gray_P } \\
\text { lane_Mean1 }\end{array}$ & $\mathrm{s}$ & $\begin{array}{l}\text { calculates the gray value moments and } \\
\text { the parameters of the approximation of } \\
\text { the gray values by a plane }\end{array}$ & 5 \\
\hline & Intensity_Mean & $s$ & $\begin{array}{l}\text { calculates the mean and the deviation of } \\
\text { the gray values in the input image within } \\
\text { Regions }\end{array}$ & 6 \\
\hline & $\begin{array}{l}\text { Fit_Surface_1st_ } \\
\text { order_Gamma }\end{array}$ & $\mathrm{s}$ & $\begin{array}{l}\text { approximates the gray values by a first } \\
\text { order surface, gamma is the most useful } \\
\text { parameter }\end{array}$ & 7 \\
\hline & Entropy & H & $\begin{array}{l}\text { calculates the entropy of gray values in } \\
\text { the image region }\end{array}$ & 8 \\
\hline & max gray & 1 & $\begin{array}{l}\text { creates the histogram of the absolute } \\
\text { frequencies of the gray values within } \\
\text { Regions in the input image Image and the } \\
\text { largest gray value }\end{array}$ & 9 \\
\hline & Fuzzy_Entropy1 & $\mathrm{H}$ & $\begin{array}{l}\text { calculates the fuzzy entropy of a fuzzy } \\
\text { set, measure of how well the image } \\
\text { approximates a white or black image }\end{array}$ & 10 \\
\hline \multirow{4}{*}{ 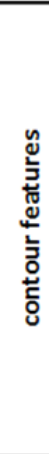 } & $\begin{array}{l}\text { runlength__ } \\
\text { features_Kfactor }\end{array}$ & B & $\begin{array}{l}\text { runlength features calculates for every } \\
\text { input region the number of runs } \\
\text { necessary for storing this region with the } \\
\text { aid of runlength coding, "K-factor" } \\
\text { indicates how much the number of runs } \\
\text { differs from the ideal of the square in } \\
\text { which this value is } 1.0 \text {. }\end{array}$ & 61 \\
\hline & $\begin{array}{l}\text { runlength_ } \\
\text { features_Lfactor }\end{array}$ & B & $\begin{array}{l}\text { L-factor indicates the mean number of } \\
\text { runs for each line index occurring in the } \\
\text { region }\end{array}$ & 62 \\
\hline & Convexity & B & $\begin{array}{l}\text { calculates the convexity of each input } \\
\text { region }\end{array}$ & 67 \\
\hline & \begin{tabular}{|l|} 
runlength_ \\
features_ \\
MeanLength \\
\end{tabular} & B & $\begin{array}{l}\text { Meanlength indicates the mean length of } \\
\text { the runs }\end{array}$ & 68 \\
\hline
\end{tabular}


the cost parameter $\mathrm{C}$ of C-SVC and the parameter $\gamma$ in the kernel function. The optimal chosen parameters $C$ and $\gamma$ are unknown before starting an optimization process. But they are very important for reaching the best classification performance for the given problem. The goal is to accurately predict unknown data, i. e. data which were not used for training. So the model selection or parameter search have to be done in a computationally efficient way.

The optimization was done on the 188 dimensional feature space. A 3-fold cross validation was used for training the LibSVM with different parameter selections to prevent the overfitting problem. The search was done by using a grid search for the different steps of parameter modification. In a first step the exponentially growing sequences of $\mathrm{C}$ and $\gamma$ as practical method was used to identify good parameters (see [17]). In the second step a fine grid search was used with an equidistant increment on the identified "better" region on the grid. Figure 4 shows the results of the optimization process.

The parameter $\gamma$ has a considerably higher influence on the total recognition rates than the parameter $\mathrm{C}$. The highest accuracy with $98.2 \%$ was reached by using $\gamma=0.05$ and $\mathrm{C}=100$. The best compromise is to use $\gamma=0.15$ and $\mathrm{C}=30$ to reach a total recognition rate of $98.1 \%$, because it is better to use a lower $\mathrm{C}$ as penalty parameter of the error term.

In the comparison of different classifiers (see Figure 5) a compromise of parameter selection was used for LibSVM.

The choice of an optimal classification algorithm is an important task of investigations in pattern classification. So some classification algorithms were tested for the given dataset by using several feature subsets, which were found with the feature selection method Info Gain. The results, total recognition rates (TRR) and recognition rates per class (RR), are shown in Figure 5 and Figure 6.

The investigation shows that the LibSVM (with TRR $=98.7 \%$ ) and the Random Forest classifier (with TRR $=97.8 \%)$ are the best classifiers for the recognition of building materials out of the plurality of tested classifiers.

In the experiments Naive Bayes reached the lowest total recognition rate $(89.3 \%)$. Better results could be observed for the J48 classifier $(96.0 \%)$ and the nearest neighbour $(97.9 \%)$.

In addition to this, only the classifiers nearest neighbour and Naive Bayes show overfitting by using an excessively complex model. They still have too many features relative to the number of given data samples.

The classwise reached individual recognition rates of the best classifier, the parameter optimized LibSVM, are explained in the following (see Figure 6). The individual recognition rates were calculated by using the different feature subsets. For all classes very good individual recognition rates over $96 \%$ was reached. The best recognition rates were reached for the class aereted concrete $(100 \%)$. Similar good recognition rates were reached for the other 4 classes in the range of $97.8 \%$ to $99.5 \%$. This performance seems to be very high but the level of complexity of the given problem has to be kept in mind. At first only 5 classes were used out of the plurality of building classes. If more classes are used in further investigations, the overall classification performance will decrease - approximately 10 percent less than the amount before.

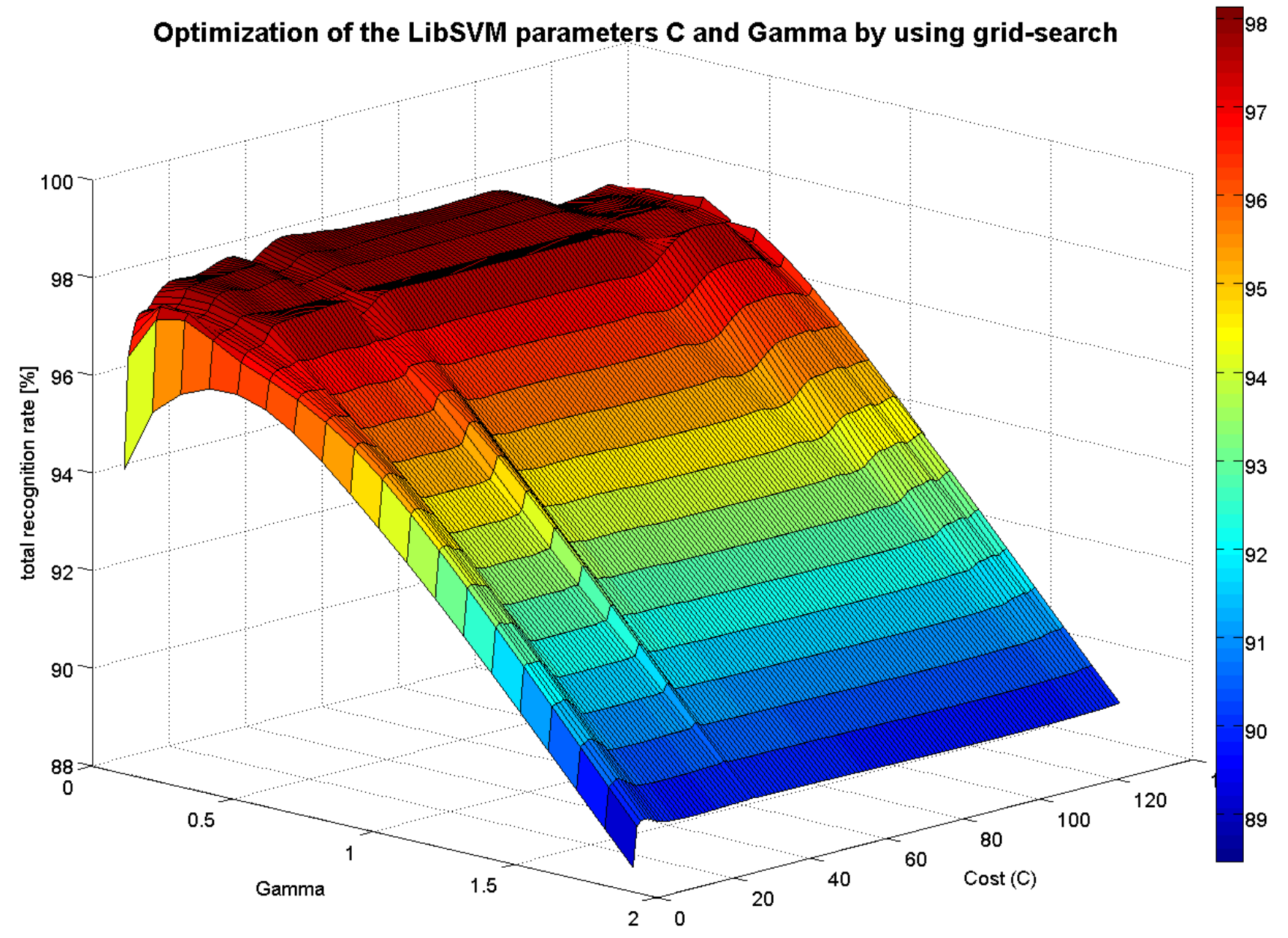

Figure 4. Surface plot of parameter optimization of LibSVM. 


\section{Classification accuracy versus the dimensionality of feature space}

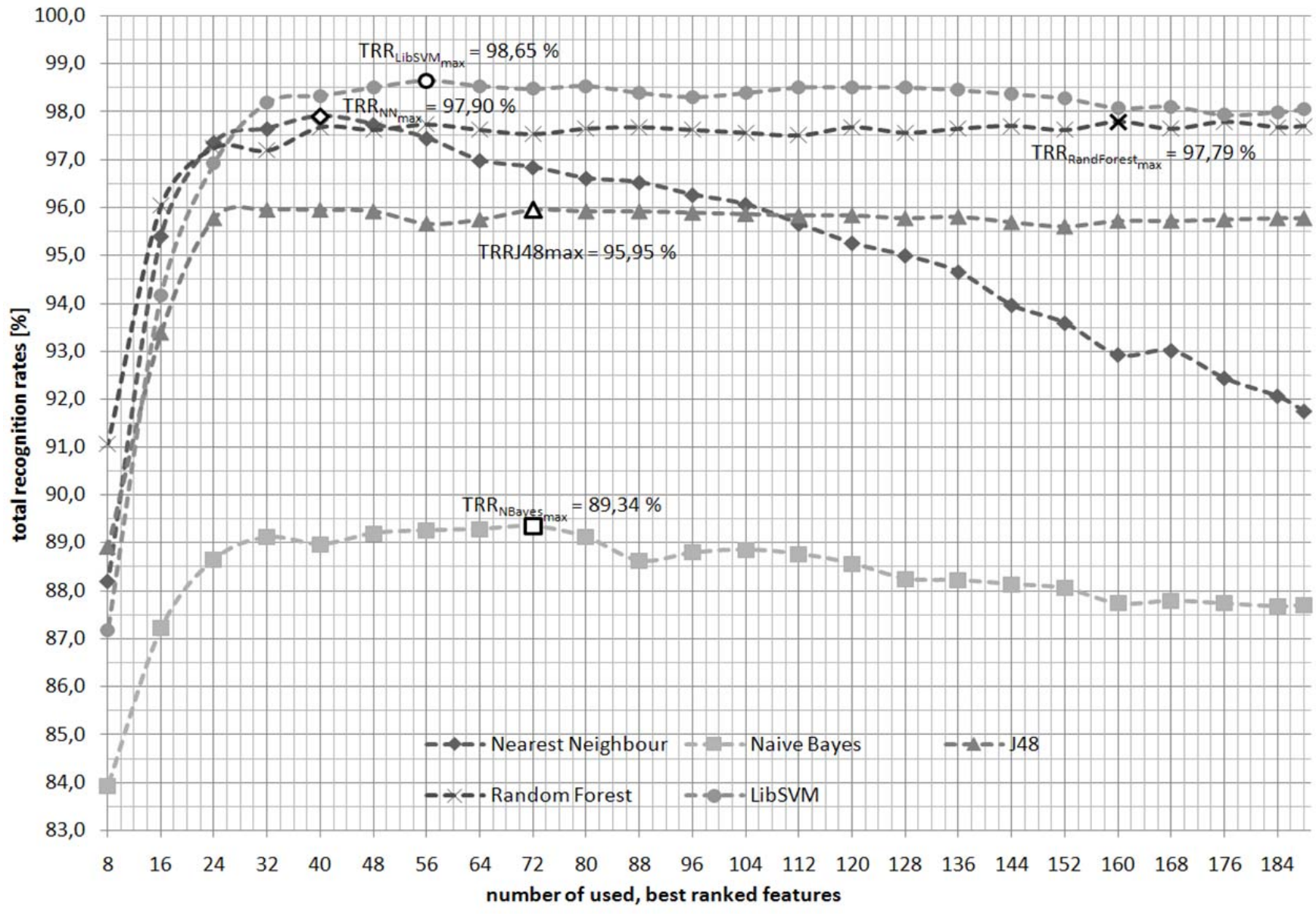

Figure 5. Classification performance of different classifiers versus dimensionality of the feature space by using the number of best ranked features.

\section{Classification accuracy of different classes versus the dimensionality} of feature space

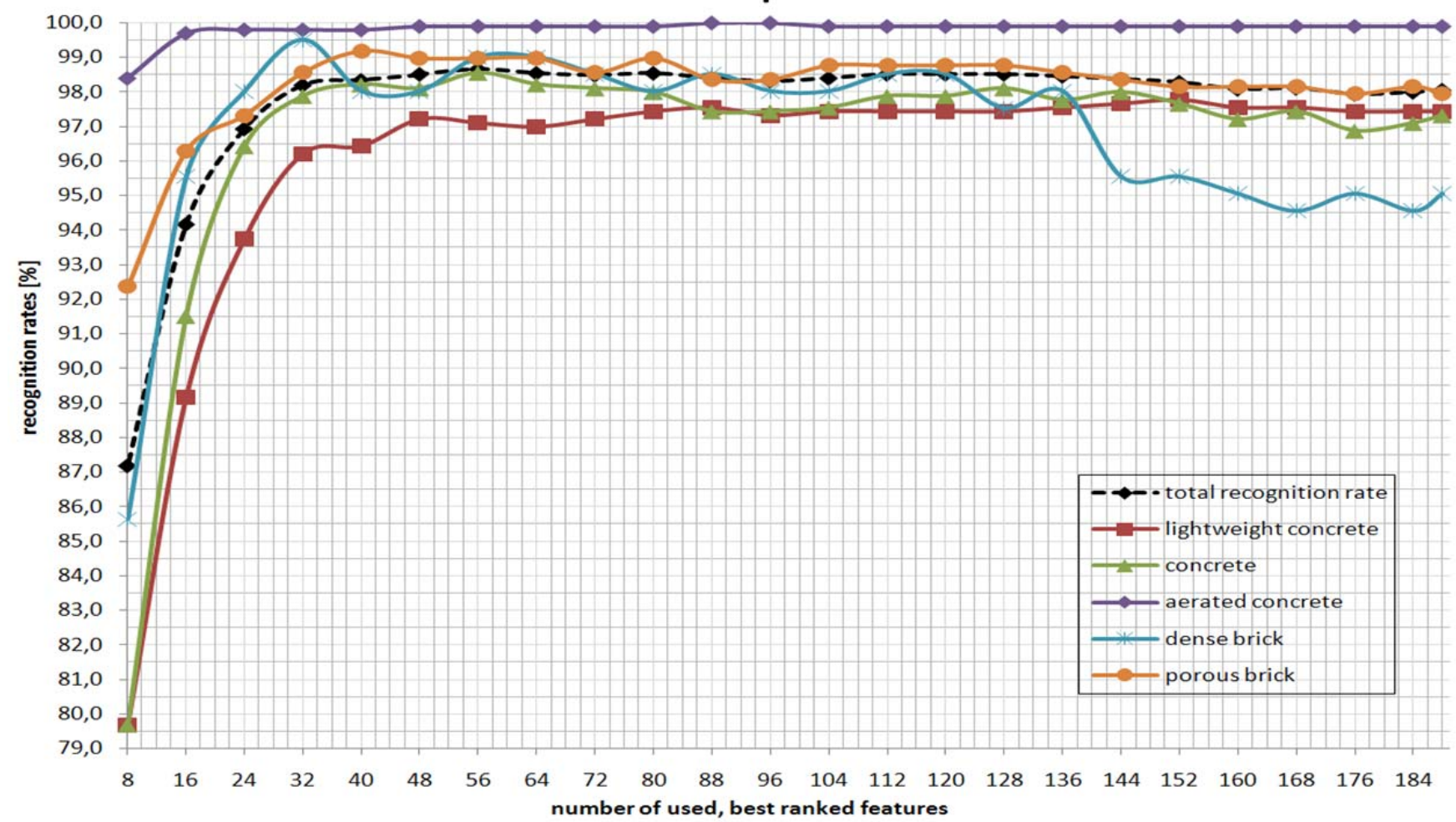

Figure 6. SVM performance versus dimensionality of the feature space by using the number of best ranked features. 


\subsection{Summary}

In this approach a part of relevant building material classes were used for testing different feature subsets and classification algorithms for the given recognition problem.

Two different filter selection methods were used for the detection of redundant and irrelevant features in the feature vector. As a result, the colour features and in particular the texture features in the $\mathrm{H}$ - and S-channel are the best discriminating features for the given problem far ahead of contour features.

After this the ranking list of features was used to build up different feature subsets with the number of best ranked features. Then different classification algorithms of the machine learning library Weka were tested, such as LibSVM, Random Forest, k-nearest neighbour, J48 and Naive Bayes by using a 10fold cross validation.

The cost parameter $C$ and the kernel parameter gamma of LibSVM were optimized to reach best classification performances and prevent overfitting. The optimization was done on the 188 dimensional feature space by using grid search. Finally it is pointed out that the parameter $\gamma$ has a considerably higher influence on the performance than the parameter $C$.

In addition the approaches demonstrated the SVM and Random Forest as the best classification algorithms for this recognition task. The parameter optimized LibSVM achieved a total recognition rate of $98.7 \%$ and the Random Forest classifier of $97.8 \%$ for the given dataset. This agrees with the fact that SVM and Random Forest are two of the most efficient classifiers today. This fact could also be demonstrated in previous investigations [6].

In future investigations the dataset has to be optimized. The dataset has to be extended for other relevant building classes.
The characteristic object features of each class and their statistical distribution have to be specified.

\section{RECOGNITION OF MINERAL AGGREGATES}

\subsection{Introduction}

Mineral aggregates which are used as concrete or asphalt additives must be analyzed according to certain European standards, in Germany also according to relevant federal standards, for example [18] in Saxony-Anhalt.

Samples from natural mineral deposits need to be taken at frequent intervals for the analysis of mineral aggregates. Certain components of aggregates can negatively affect the mechanical strength of produced concrete or asphalt as well as cause the destructive alkali silica reaction, which is very harmful and costintensive. A method for the automatic inspection of mineral aggregates was developed for analyzing aggregate samples with a weight of nearly $1 \mathrm{~kg}$ to overcome these problems.

\subsection{Hardware and dataset}

For the image based recognition of mineral aggregates a specialized system for feeding, separation and image acquisition was developed (Figure 7). This device permits the handling of samples with a component size from 4 to $32 \mathrm{~mm}$ in diameter. The sample is filled in the charging bin, gets separated via $\mathrm{V}$ profiled chute and tracks over a belt conveyor.

The aim is to get non-touching and non-overlapping objects in the field of view of the color linescan camera, resulting in single object images after segmentation. The whole segmentation process is carried out line-wise on the data stream from the color line-scan camera. This is done in real time.

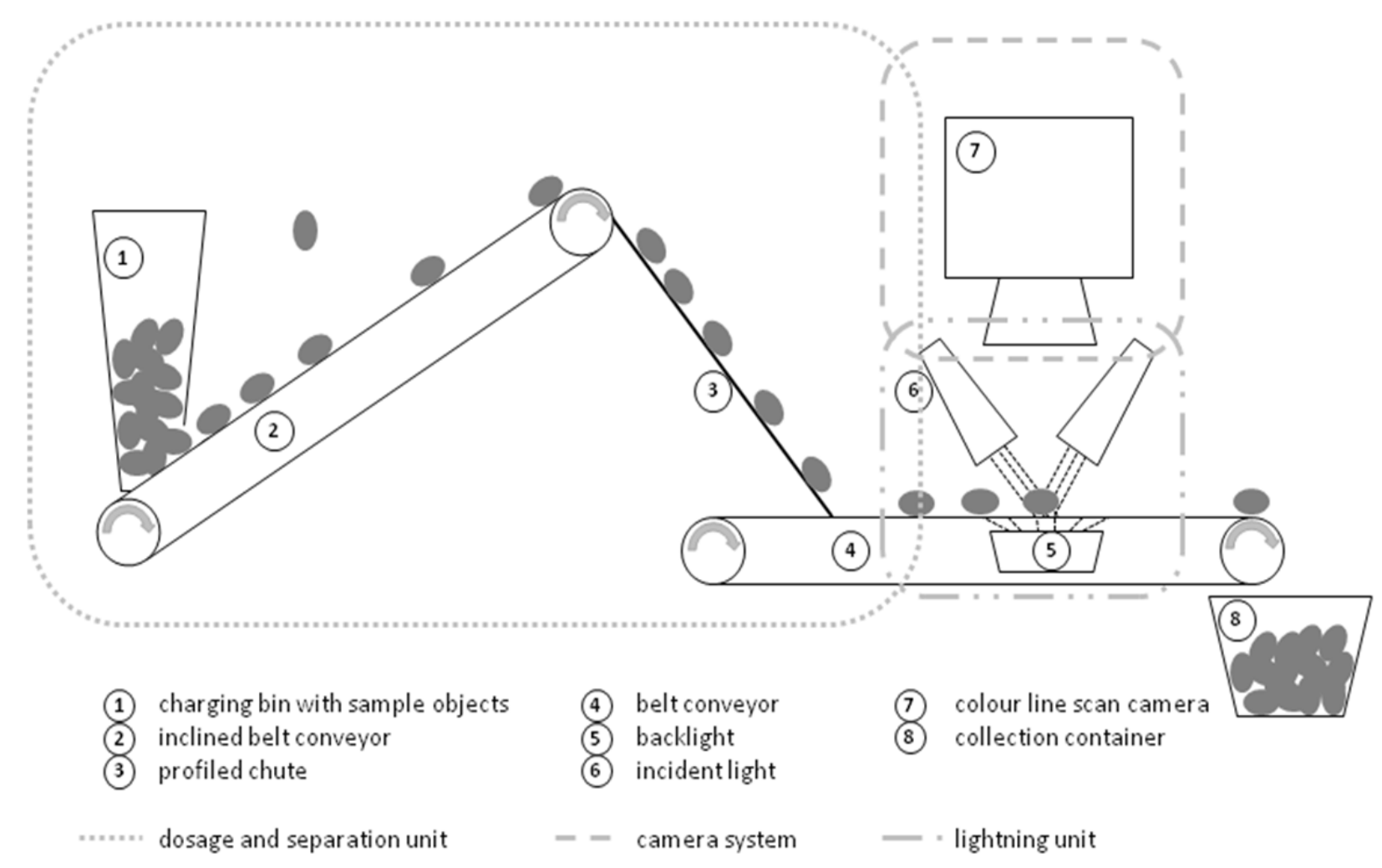

Figure 7. Hardware setup for image acquisition of mineral aggregates. 


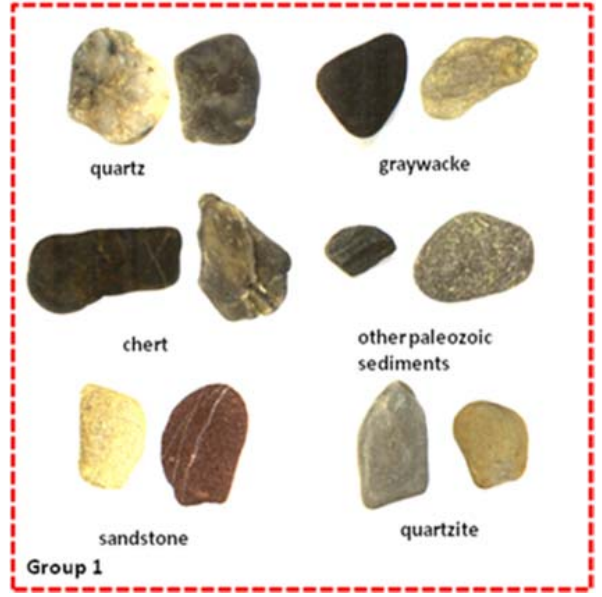

Figure 8. Components of mineral aggregates.

To achieve higher data rates it is possible to port this processing to an FPGA. This could also be a good solution in conjunction with a higher image resolution which increases the amount of data. The experiments described in this paper are done with a color line-scan camera with 2048 pixels.

By using this setup two datasets with different sample objects were generated, a dataset with 16,000 objects (dataset 1 - DS1) for parameter optimization and classifier training and an independent test dataset with nearly 3,000 objects (dataset $2-$ DS2). To cover also effects from a possible slightly unstable illumination both datasets were acquired on different days. All images are stored in loose-less Windows-Bitmap-format (BMP). Some sample objects are shown in Figure 9.

The different components of mineral aggregates need to be grouped according to the standard described in [18]. Group 1 consists of harmless and the groups 2 till 3 of harmful components.

There are three main difficulties for the given recognition problem:

- Very high variability within the sub-classes

- Very low variability over all classes

- Imbalanced dataset: especially group 3 consists of very rare objects

So only the 4 superordinated groups 1 to 4 were used for training and testing (see Figure 8 and Figure 9).

Handling these problems needs a very accurate feature selection and parameter optimization.

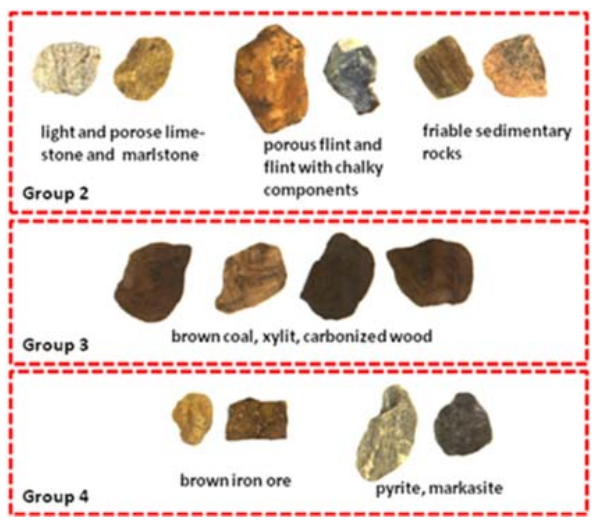

Figure 9. Particular components of mineral aggregates to be considered.
Distribution of the Information Gain Score for the feature set

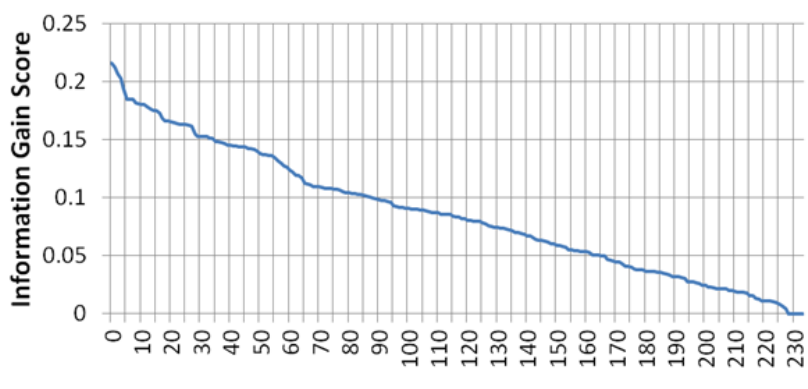

rank of the feature according to the score value

Figure 10. Feature scoring.

\subsection{Feature selection}

Our feature pool consists of standard image processing operators from the library Halcon [1]. Nearly all used features are invariant against the Euclidean transformations rotation, translation and scaling.

For feature selection the Information Gain score was used [3]. Dataset 1 was separated in two sub-sets DS1.1 (66\%) and DS1.2 (34\%) to determine the most relevant features. The feature scoring was calculated on DS1.1. The features are ordered in ascending order according to the score. For reasons of visualisation there are only numbers drawn on the $\mathrm{x}$-axis in Figure 10. It shows that there are no clearly strong features with a significantly higher score than others. The score values are relatively low, which results from the very high intra-classvariability of the given dataset.

Further considerations needed to be done because the curve in Figure 10 did not give a clue how to set the relevance threshold. The chance for overfitting is very high, especially for the given, very complex recognition problem and a feature set with 235 possible useful features.

So the following scheme was conducted to find the most relevant features with training dataset (DS1.1) and test dataset (DS1.2):

1) Calculate the feature score on the training dataset

2) Sort the features in ascending order

3) Repeat the following until the feature set is empty:

4) Train a classifier on the training dataset

5) Evaluate the classifier accuracy on the test dataset

6) Remove the $\mathrm{N}$ lowest scored features

7) Plot the classifier accuracy against the feature set size

8) Choose the optimal feature set size

To handle imbalanced datasets it is very important to use a performance measure which is independent of the number of objects per class. The total recognition TR calculated as follows doesn't achieve this:

$\mathrm{TR}=\frac{\text { number of correctlyclassifiedinstances }}{\text { number of allinstances }}$. 


\section{dimensionality of the feature space vs. accuracy for J.48}

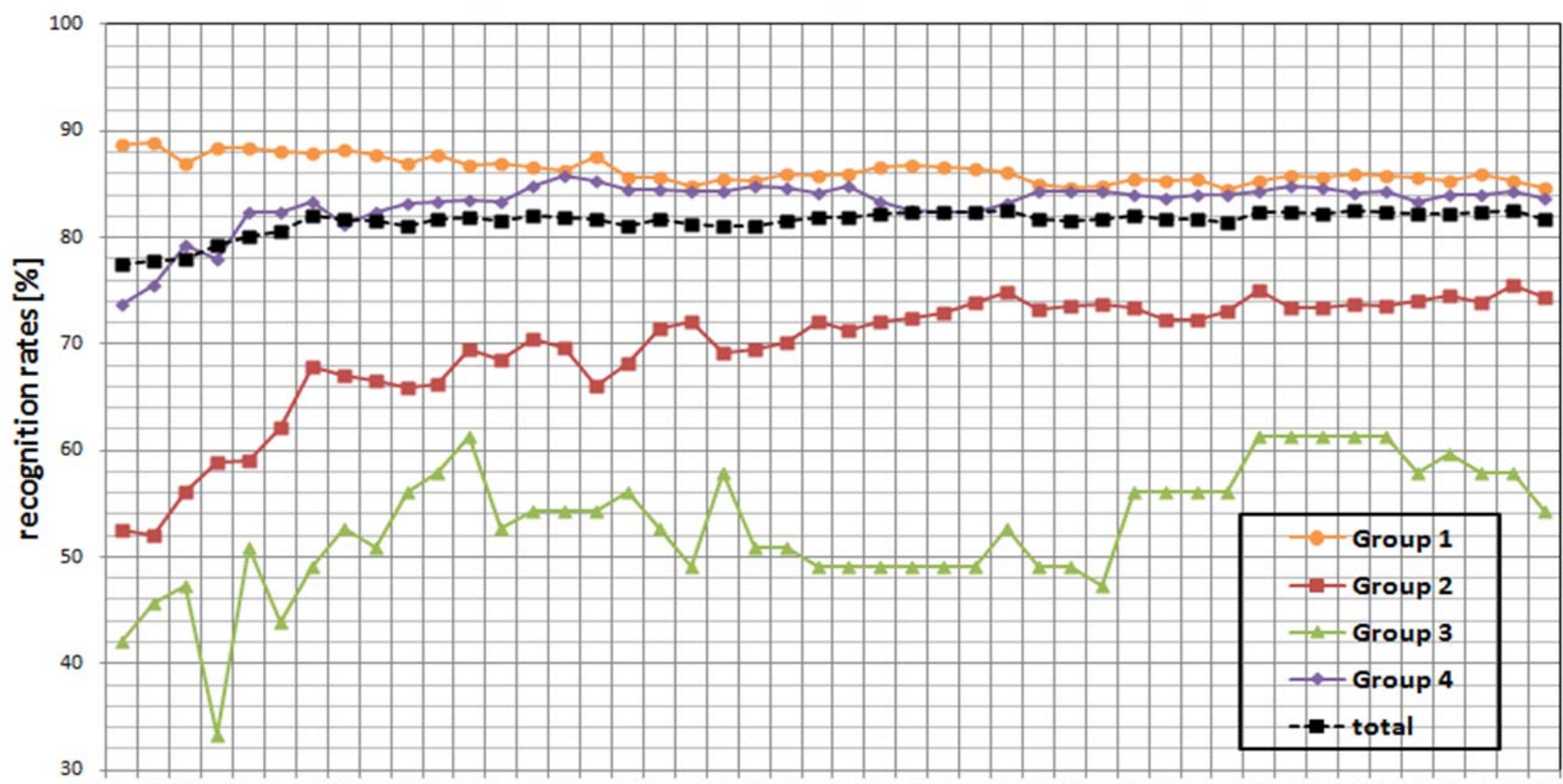

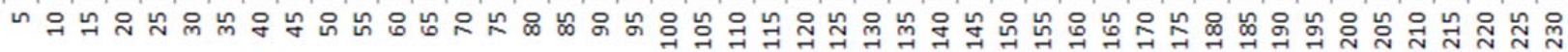
number of used, best ranked features

Figure 11. Filter-based feature selection.

In conjunction the balanced error rate (BER) is calculated as the mean of the recognition rates for the single classes and is independent of the number of instances per class.

Applying the described scheme we can plot the accuracy versus the dimensionality of the feature space (see Figure 11). In this way the optimal feature subset was chosen with the 145 most relevant features.

It turned out that the most relevant features for this task are

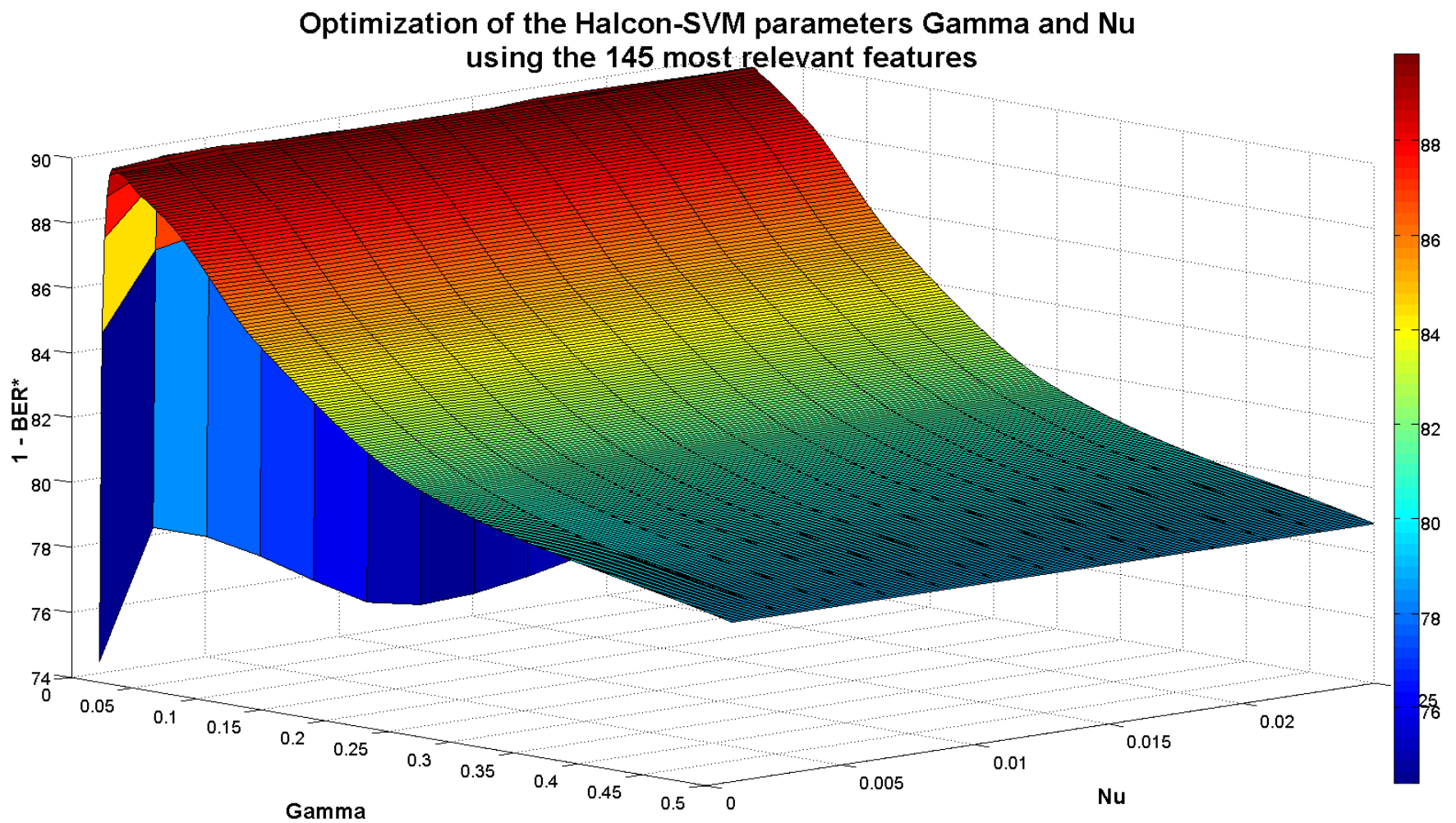

Figure 12 Results from the grid search algorithm. 


\section{Balanced error rate vs. Recognition rate}

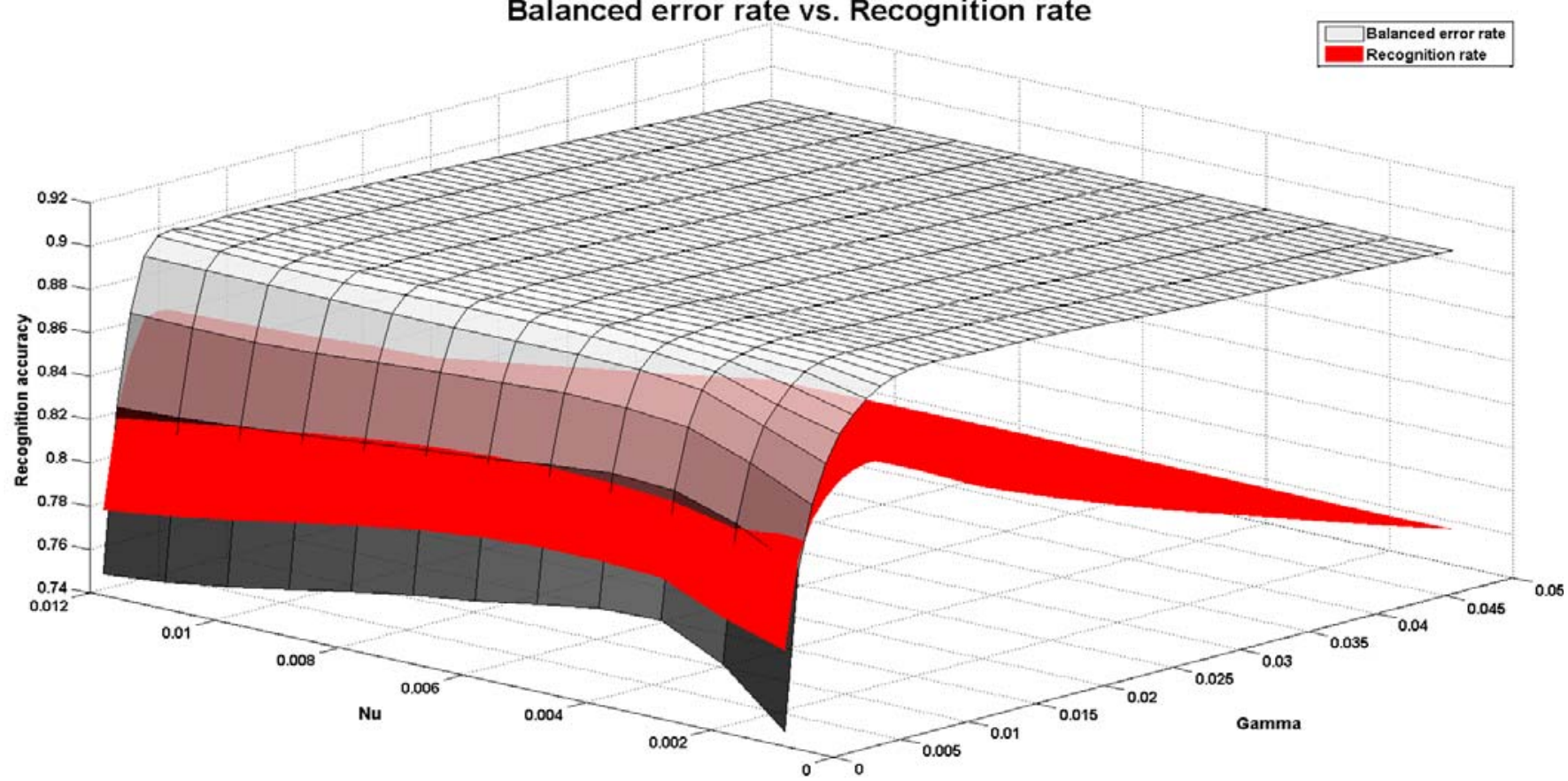

Figure 14. Balanced error rate vs. total recognition rate (top view).

gray value mean and deviation in the hue and saturation channel of the HSI color space as well as entropy and anisotropy, features derived from the co-occurrence matrix and Laws texture features. As the optimal number of features has been found we are facing the problem to find the optimal values for the support vector machine. For this task a Nu-SVM was used. The regularization parameter $\mathrm{Nu}$ is more intuitive than the Cost parameter C of the C-SVM. It can be seen as an upper border on the training set error and a lower border on the amount of training vectors which become support vectors.

\subsection{Parameter Optimization}

For our research we mainly used the radial basis function kernel (rbf). The kernel parameter Gamma $(\gamma)$ and the regularization parameter $\mathrm{Nu}$ of the SVM need to be chosen with care. To find an optimal parameter set a gridsearch method with 3-fold-crossvalidation on the training-set (DS1.1)

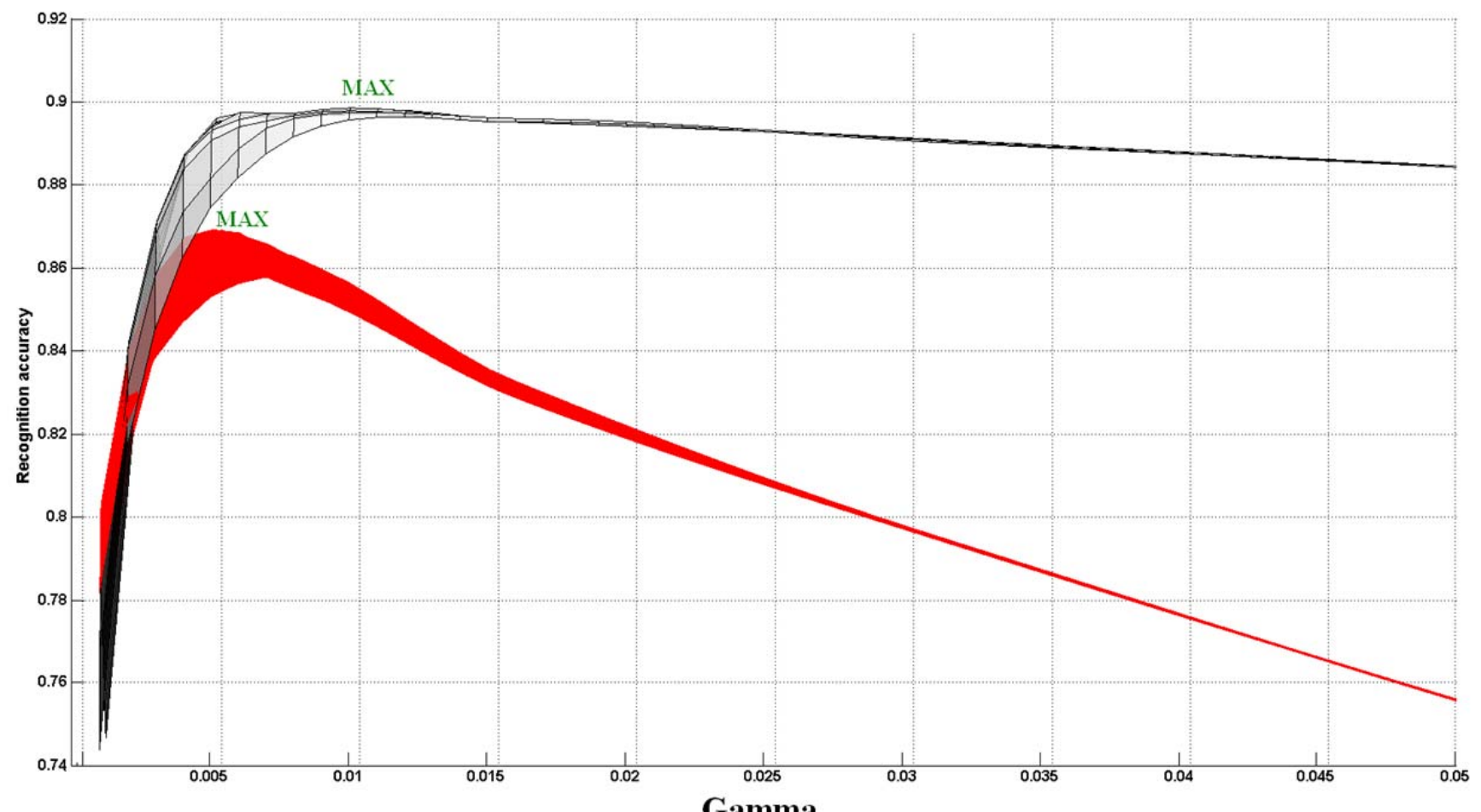

Figure 13. Balanced error rate vs. total recognition rate (side view) with tagged global maxima. 


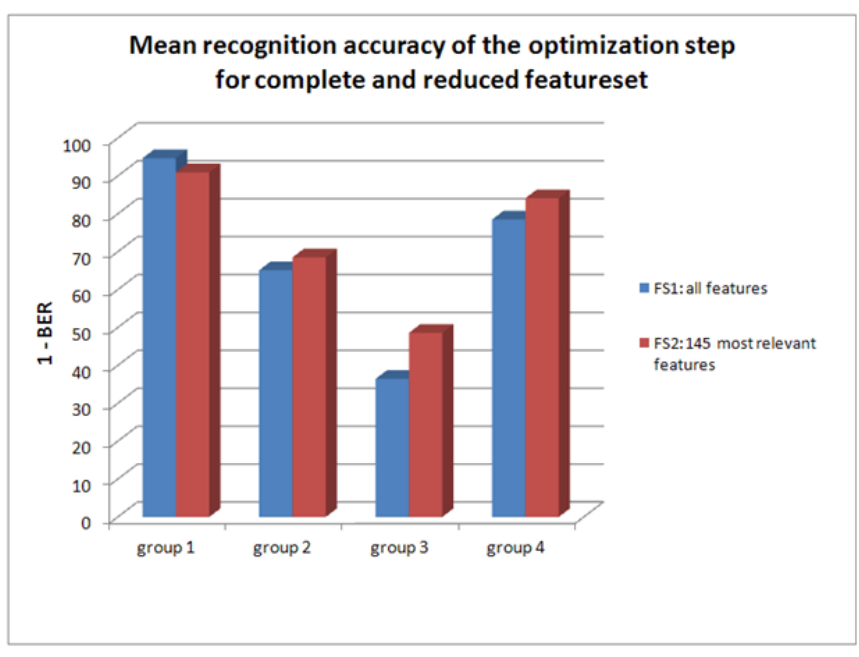

Figure 15. Mean accuracy of different parameter combinations during the optimization for FS1 and FS2.

with nearly 16,000 objects was also conducted. So the optimal parameter combination for the final SVM could be estimated.

The position of the grid points in the intervall $\left[0\right.$, GridMax $_{\mathrm{Nu}}$, Gamma $]$ are calculated according to

$$
\operatorname{Pos}_{N u, \text { Gamma }}=\operatorname{GridMax}_{\mathrm{Nu}, \mathrm{Gamma}} / 2^{i-n+1}
$$

with $0 \leq \mathrm{i} \leq 9$ and $\mathrm{n}=10$

In this way 100 grid points $(\mathrm{Nu}, \mathrm{Gamma}$ ) were evaluated. The best parameter combination is chosen for the final classifier.

Figure 12 shows that the performance of the classifier mostly depends on the value of Gamma. A higher Gamma results in a dissemination of the influence on the decision boundary over the whole training dataset and thus also less relevant training samples have a great impact on the decision boundary. The classifier learns the data at heart.

The total recognition rates as well as the balanced error rate during the grid search were calculated to illustrate the problem of imbalanced data for parameter optimization. Figure 14 shows that the highest accuracy can be found with different values depending on the optimization criterion. The impact of classes with less available objects is negatively affected by using the total recognition rate.

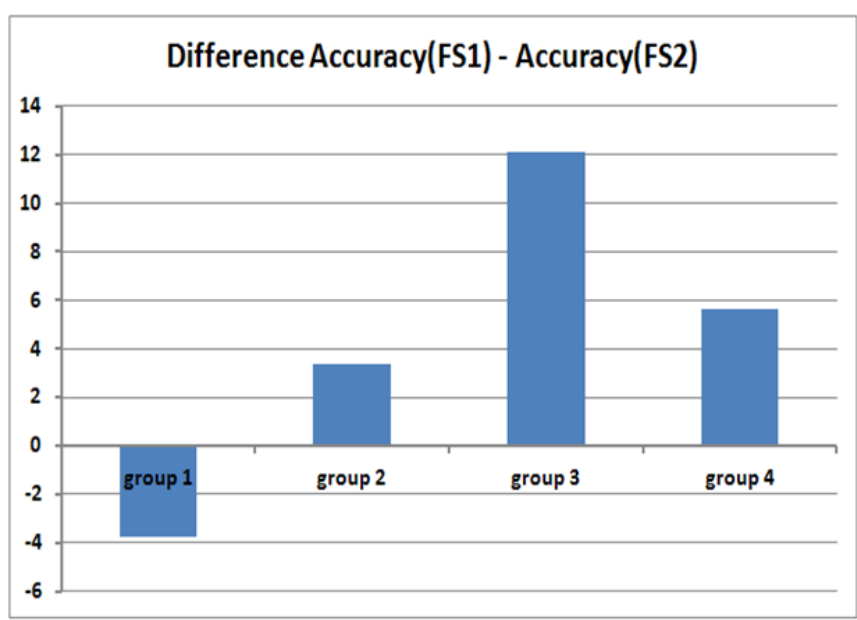

Figure 16. Differences in accuracy between FS1 and FS2.

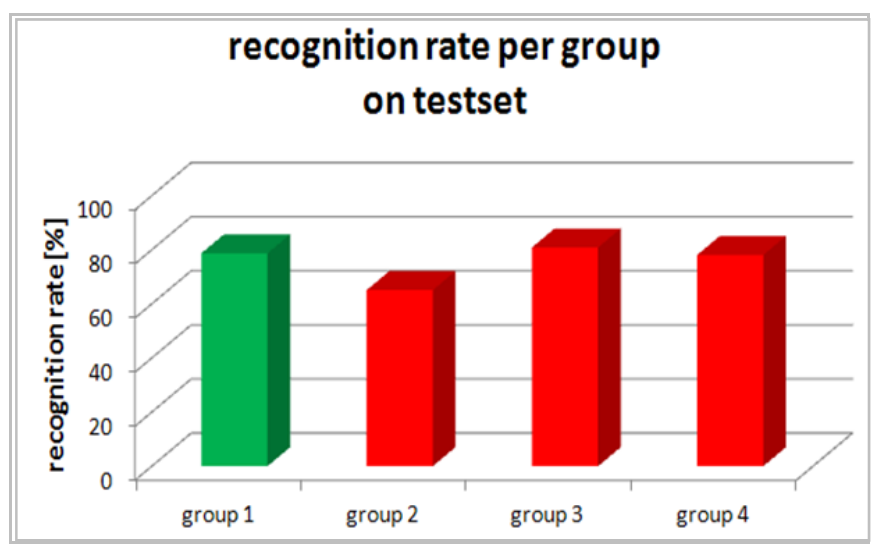

Figure 17. Final results on the test dataset.

The classification accuracy was calculated with the different parameter combinations of $\mathrm{Nu}$ and Gamma and also on the whole feature set to illustrate the effect of the feature selection. In Figure 15 the mean accuracy is shown, which is achieved by using cross-validation on the training dataset DS1.1. FS1 is the feature set with all features and FS2 with the 145 most relevant features. A high increase of the accuracy for the underrepresented groups 2 and 3 could be reached by using the balanced error rate.

The algorithms were tested on an independent test dataset to estimate the performance of the whole method.

\subsection{Algorithm Test}

The test of whole recognition system consisting of the hardware setup and the developed software in conjunction with the optimized classifier models was the last step of our research. The optimized classifier was trained on the whole DS 1 (DS 1.1 and DS 1.2). Therefore an independent test dataset DS2 was acquired and the recognition rates for all four groups were estimated. The result is shown in Figure 17.

\subsection{Summary}

In this chapter a method to analyze mineral aggregates is presented. A recognition rate of $76 \%$ could be achieved, which turned out to be a very good result for this complex task with very high intra-class-variability and low inter-class-variability. It turned out that grouping the different components of mineral aggregates in the feature space and training a classifier only with four superordinated groups resulted in a lower performance than training a classifier on all 16 sub-groups and aggregate the class labels to the four groups [20]. This can be explained by the fact that the grouping is been done on the basis of the effect of the component on the produced concrete and not on the phenotypical appearence.

\section{COMPARISON OF BOTH RECOGNITION PROBLEMS IN TERMS OF COMPLEXITY}

A principal component analysis (PCA) and a visualisation of object clusters in feature space was realised with Matlab ${ }^{\circledR}$ for obtaining a better understanding of the two given recognition problems and the separability of the classes by using the feature vectors with all calculated features. The data of the CDW and the mineral aggregate are given as feature vectors. They build clusters in the $p$-dimensional cartesian coordinate system. The 


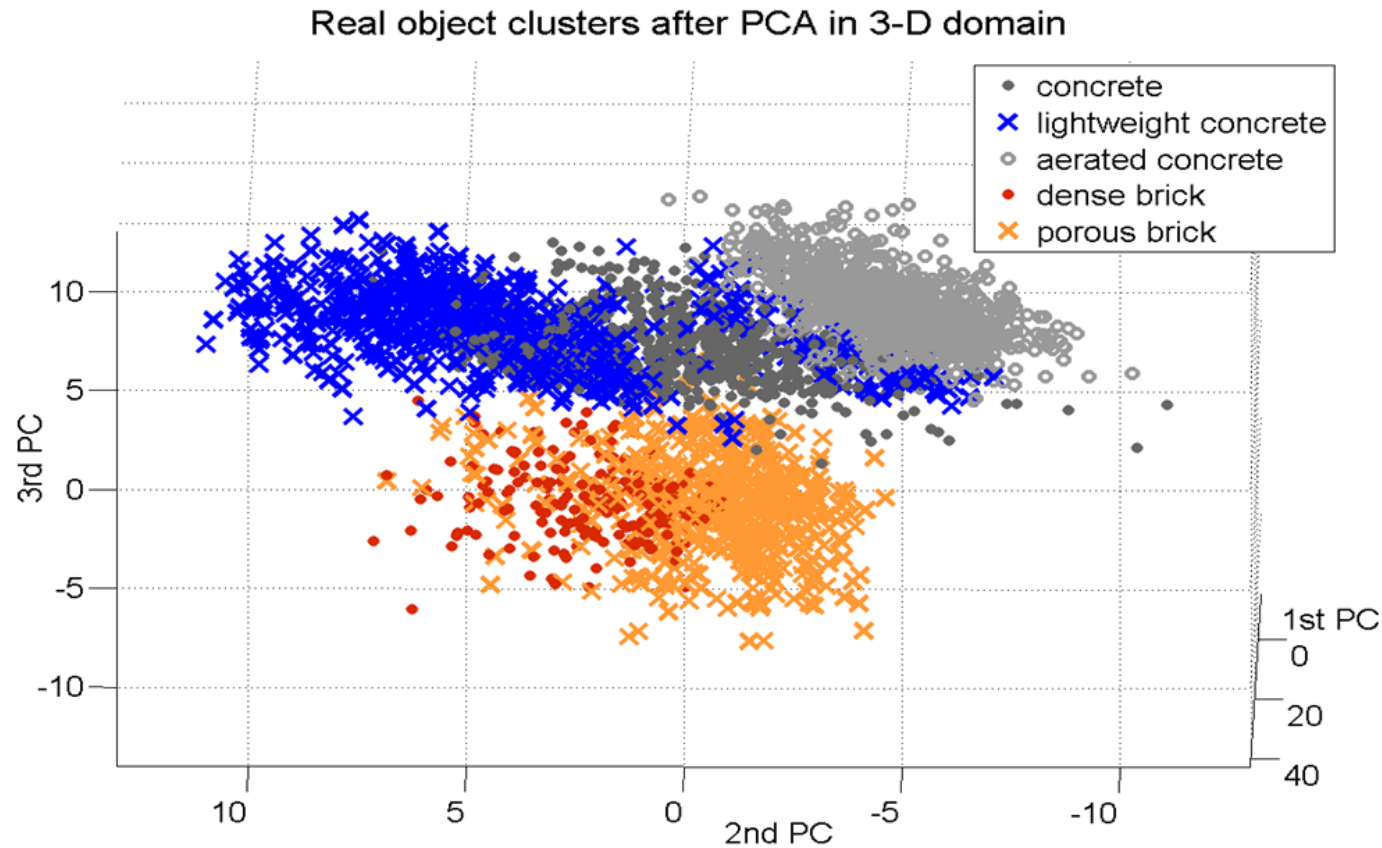

Figure 19. CDW aggregates - real cluster representation after PCA in 3D-domain.

Clusters cannot be visualised in the $p$ - dimensional feature space and so the characteristic and complexity of the given recognition problem cannot be evaluated. The principal component analysis (PCA) allows the visualisation of the given data based upon a reduction of the relevant information in data by using only the first three principal components with the highest variance. The goal of the PCA is the approximation of the $p$ features by a smaller number of meaningful linear combinations (principal components).

The visualisation of the first three principal components of the $\mathrm{CDW}$ and the mineral aggregate recognition problem are shown in Figure 19 and Figure 18. The only visualisation of the first three principal components is always connected with a loss of information for complex high dimensional data.

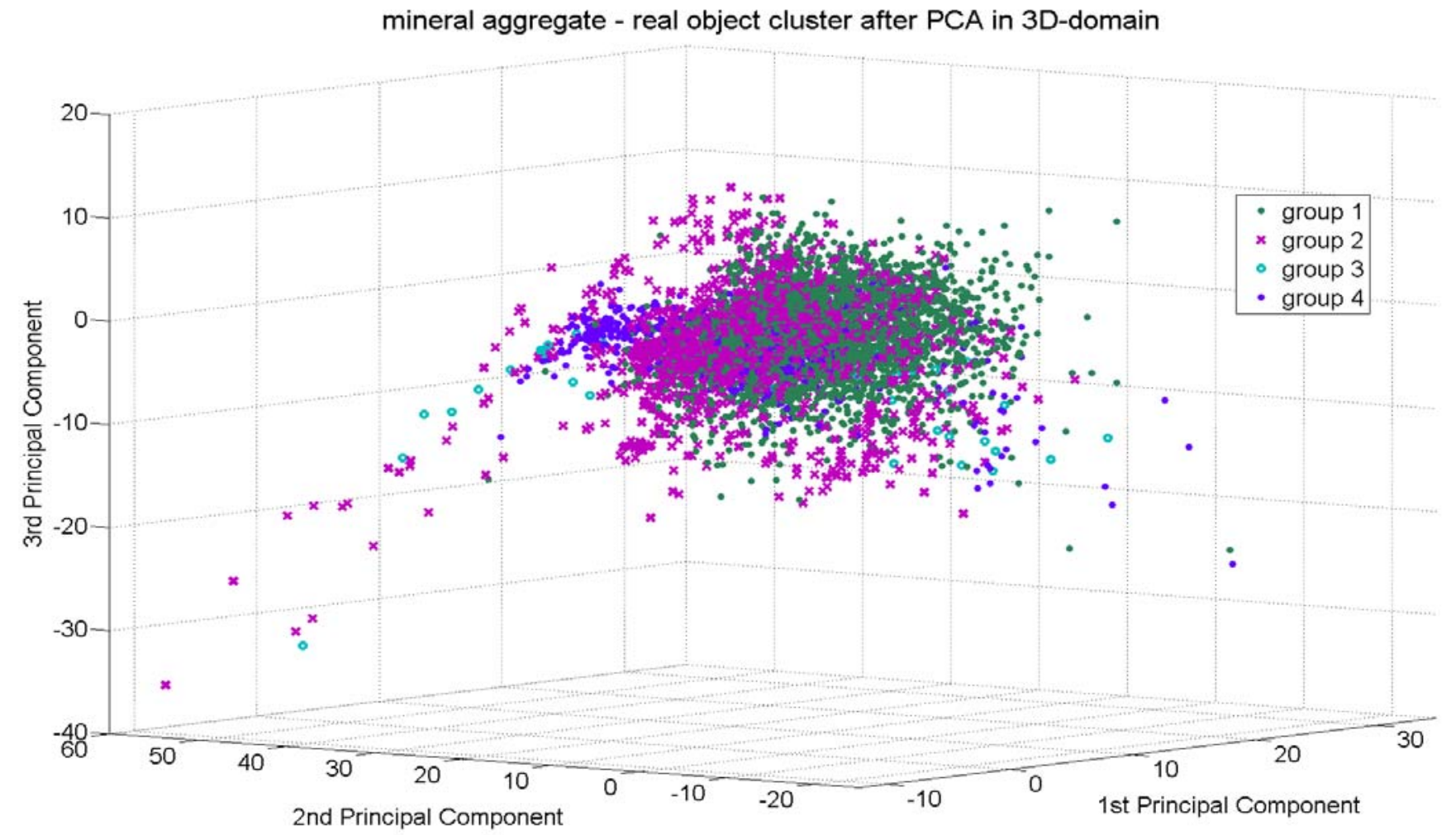

Figure 18. Mineral aggregates - real cluster representation after PCA in 3D-domain. 
Nevertheless it allows the comparison of the underlying complexity between different recognition problems, especially in consideration of the calculated total variance of the first three principle components.

In Figure 19 strong distinctions between types of brick and types of concrete are well visible in the distance between the related clusters. But also the classes of concrete and the classes of brick are well-defined among themselves. The calculated total variance of the first three principal components is only $39.3 \%$ but the clusters of the given data can already be separated in this feature space.

In Figure 18 no homogeneous and well-defined clusters can be found for the classes of mineral aggregates. All four superordinated classes show a wide-ranging overlapping so no class boundaries in this space can be found. The calculated total variance of the first three principal components here is $44.3 \%$ but the clusters of the given data cannot be separated in this feature space.

In summary, it becomes clear that the recognition task of the given sub-classes of mineral aggregates has a higher complexity than the recognition task of the given classes of CDW aggregates. If the amount of the implemented CDW classes increases, the complexity will also increase. But it is believed that the complexity of the CDW aggregate will always be lower than the complexity of the mineral aggregate because of the lower intra-class-variability and the higher inter-class-variability of CDW classes.

\section{CONCLUSIONS}

As described in the paper both problems can be solved by intelligent image processing algorithms. The recognition of CDW is possible with a higher accuracy. On the one hand the achieved performance seems to be adequate for an automated sorting device. On the other hand it turned out that the accuracy for the recognition of components of mineral aggregates needs to be improved to get a practical solution. This can be done by using features from the near infrared domain (NIR). Otherwise the effort for a manual follow-up checking is too high.

For the analysis of components of mineral aggregates an automated image acquisition system could be developed. The image acquisition for the recognition of CDW was done with manual placing the objects in the field of view of an area scan camera. But it turned out that the algorithms could be integrated into sorting devices. We also need to keep in mind that the research in the field of CDW recognition was done on a subset of the possible classes. In further research also objects from the excluded classes need to be collected and analysed.

\section{ACKNOWLEDGEMENTS}

The project "Automatic Petrographic Inspection" funded by the German Federal Ministry of Economics and Technology forms the basis of this paper. The responsibility for the content of this paper lies with the author.

A comprehensive hand sorted sample material of mineral aggregate builds the basis of one of the used image datasets. This comprehensive task was realized by experts of the german inspection organization PST Bernburg, Germany. Our special thanks go to Mrs. Kallies and her colleagues for the supply of the material samples.

\section{REFERENCES}

[1] Halcon, "Reference Manual - Halcon 8.0.3", MVTec Software $\mathrm{GmbH}$, www.mvtec.com, 2009.

[2] I. Guyon, A. Elisseeff, "Feature extraction: foundations and applications", Berlin, Springer Verlag, 2006.

[3] T. M. Mitchell, "Machine Learning". The Mc-Graw-Hill Companies Inc, 1997.

[4] R. Setino, H. Liu, "Chi" Feature selection and discretization of numeric attributes", In: Proceedings of the Seventh IEEE International Conference on Tools with Artificial Intelligence, 1995.

[5] R. O. Duda, P. E. Hart, D. G. Stork, "Pattern Classification, A Wiley Publication, 2001.

[6] K. Anding, "Automatic Quality Assurance of Wheat by Using Supervised Machine Learning Methods in Image Processing (Automatisierte Qualitätssicherung von Getreide mit überwachten Lernverfahren in der Bildverarbeitung)", dissertation, Ilmenau University of Technology, 2010.

[7] M. Hall, F. Eibe, G. Holmes, B. Pfahringer, P. Reutemann, I. H. Witten, "The WEKA Data Mining Software: An Update", SIGKDD Explorations, vol. 11(1), 2009.

[8] B. Boser, I. Guyon, V. Vapnik, "A Training Algorithm for Optimal Margin Classifiers", Proceedings of the Fifth Annual Workshop on Computational Learning, 1992.

[9] V. Vapnik, "The Nature of Statistical Learning Theory", New York, Springer Verlag, 1995.

[10] Monitoring of Building Waste 2008 (Mineralische Bauabfälle Monitoring 2008), society for the recycling economy in the building sector (Arbeitsgemeinschaft Kreislaufwirtschaft Bau KWB), Berlin, 2011.

[11] C. F. Hendriks, "The building cycle", Aeneas, Netherlands, 2000.

[12] A. Mueller, "Closed loop of concrete rubble? Lecture notes", Bauhaus-University of Weimar. available in: www.uniweimar.de/Bauing /aufber/Lehre/ Gastvorlesung/Barcelona/Lecture07.pdf, 2007.

[13] RECDEMO: "Complete utilization of the sand fraction from demolition waste recycling”, Laymann report. EU LIFE, 2004.

[14] M. Dehler, "Optical Sorting of Mineral Commodities (Optische Sortierung von mineralischen Rohstoffen)", Onlinefassung, 2003.

[15] COMMODAS, "We sort it out", Catalog. 7. http:// www.commodas.de, 2007.

[16] Chih-Chung Chang, Chih-Jen Lin, "LIBSVM : a library for support vector machines". ACM Transactions on Intelligent Systems and Technology, 2:27:1--27:27, 2011. Software available at http://www.csie.ntu.edu.tw/ cjlin/libsvm.

[17] Chih-Wei Hsu, Chih-Chung Chang, Chih-Jen Lin, "A Practical Guide to Support Vector Classification", Department of Computer Science National Taiwan University, http:// www.csie.ntu.edu.tw/ cjlin, 2010.

[18] ZTV-StB LBB LSA 09/10 - Zusätzliche Technische Vertragsbedingungen und Richtlinien für Straßenbau-arbeiten für den Geschäftsbereich des Landes-betriebes Bau Sachsen-Anhalt. 2009.

[19] QI Inspector: ergonomic vision and installation workstation with colour camera (QI Inspector: Ergonomischer Sicht- und Montagearbeitsplatz mit Farbkamera), available in: http://www.stz-ilmenau.de/products/, 2011.

[20] G. Linß, K. Anding, D. Garten, M. Rückwardt: “Automatic Petrographic Inspection by Using Image Processing and Machine Learning”, XX IMEKO World Congress, Metrology for Green Growth, Busan, Korea, 2012. 